\title{
Envelope Correlation Parameter Measurements in a MIMO Antenna Array Configuration
}

\author{
Constantinos Votis, George Tatsis, Panos Kostarakis \\ Physics Department, University of Ioannina, Ioannina, Greece \\ Email:kvotis@grads.uoi.gr,gtatsis@grads.uoi.gr,kostarakis@uoi.gr \\ Received January 11, 2010; revised February 12, 2010; accepted March 15, 2010
}

\begin{abstract}
In a $2 \times 2$ MIMO antenna array system envelope correlation coefficient " $\rho$ " shows the influence of different propagation paths of the RF signals that reach the antenna elements. The approximated value of this coefficient is based on a simple closed-form equation and also varies from 0 to 1 . Quite perfect performance for MIMO applications is achieved when this parameter approximates to zero. In this paper, we evaluate an antenna diversity MIMO system by measuring the envelope correlation coefficient. The corresponding results in our antenna array configurations show that the measured " $\rho$ " has very small values and approximates to zero. This observation indicates quite perfect behavior and performance of our MIMO antenna array system.
\end{abstract}

Keywords: Scattering Parameters, Envelope Correlation, Printed Dipole Antenna

\section{Introduction}

Multiple Input Multiple Output (MIMO) systems have received a great attention, recently. This architecture uses more than one antenna elements in transmitter and receiver ends and is able to overcome the limit of channel capacity in a rich multipath environment [1]. The theoretical capacity of the system increases linearly with the number of elements in MIMO antenna arrays. However, practical considerations indicate that the corresponding capacity of the system may be reduced if the received signals in any of the different antenna elements are correlated [2]. This effect proposes that diversity gain is obtained in the antenna system when the value of " $\rho$ " is less than 0.5 [3]. It is obvious that correlation affects MIMO performance and represents a crucial parameter for modern wireless applications [4].

Moreover, MIMO design considerations include these antenna diversity techniques that also increase spectrum efficiency. It is also recognized that mutual coupling of the antenna degrades the performance of these systems.

These observations and an amount of corresponding research activities indicate that MIMO system performance is a crucial topic and for this investigation some parameters need to be considered. The envelope correlation between antenna elements is one of most important because it is related with the spectral efficiency and may provide degradation on performance of these applications.
Antenna correlation calculation procedure is provided by appropriate methods of analysis. Basically, three methods are used for these envelope correlation coefficient calculations. One of them is based on the far-filed radiation pattern. However, it is a time-consuming process $[5,6]$. This requires the corresponding numerical or experimental analysis and therefore is a cumbersome process. The second method is based on Clarke's formula [7] and has recently been used $[8,9]$. The third method is suitable for experimental measurements and requires the knowledge of scattering parameters obtained on the antenna elements. This last method is the one we adopted throughout this paper. The procedure of calculating the correlation between antennas in a two - antenna system using the scattering parameters is proposed in [10]. In our study, envelope correlation of eight antenna array types are presented and investigated for two indoor environments.

The present paper is structured as follows: in Section 2, the basic theoretical background is presented; the proposed antenna array implementation aspects are introduced in Section 3. Antenna array configurations are investigated in terms of envelope correlation and the corresponding results are discussed in Section 4. The experimental observations are summarized in Section 5.

\section{Theory}

The method of calculating envelope correlation of ele- 
ments in each antenna array configuration is based on a fundamental Equation (1) that requires 3-dimensional radiation pattern considerations.

$$
\rho_{e}=\frac{\left|\iint_{4 \pi}\left[\vec{F}_{1}(\theta, \phi) \cdot \vec{F}_{2}^{*}(\theta, \phi)\right] d \Omega\right|^{2}}{\iint_{4 \pi}\left|\overrightarrow{F_{1}}(\theta, \phi)\right|^{2} d \Omega \iint_{4 \pi}\left|\overrightarrow{F_{2}}(\theta, \phi)\right|^{2} d \Omega}
$$

The parameter $\vec{F}_{1}(\theta, \phi)$ is the field radiation pattern of the antenna system when only the port $i$ is excited and all other ports are terminated to $50 \Omega$ load. The symbol - also denotes the Hermitian product $[4,10]$.

Recent research activities have shown that the envelope correlation can be well defined by a simple closedform equation that relates the scattering parameters of the elements in an antenna array configuration. Especially, in case of a multipath indoor environment with a uniform distribution of Equation (2) is proved to be a good approximation [4]. For two antenna elements this equation using the scattering parameters becomes:

$$
\rho_{e}=\frac{\left|S_{11}{ }^{*} S_{12}+S_{21}{ }^{*} S_{22}\right|^{2}}{\left(1-\left|S_{11}\right|^{2}-\left|S_{21}\right|^{2}\right)\left(1-\left|S_{22}\right|^{2}-\left|S_{12}\right|^{2}\right)}
$$

It is obvious that radiation pattern in Equation (1) makes the calculation more complicated than the envelope correlation calculations based on in Equation (2). The practical advantage of the third method that is based on second equation is that not only is quite simple to use it experimentally, but also provides sufficiently accurate results in many experimental environments such as indoor environments with rich multipath propagation performance.

\section{Antenna Array Aspects}

The mathematical consideration given by in Equation (2) is related to the corresponding antenna array structure that is comprised by two identical printed dipole antennas with integrated balun and a plane reflector of aluminum. Figure 1 presents the layout of antenna dipole. Geometry parameters of the printed dipole have been further studied and investigated [11-13]. Its characteristics are summarized in Table 1. Each of the two identical printed dipoles has a resonance point close to the frequency range of $2.4 \mathrm{GHz}$ and the corresponding resonance bandwidth is quite $500 \mathrm{MHz}$. In addition, the reflector backplane (Figure 2) is designed and implemented to allow the positioning of the antenna elements in various configurations. From these considerations it is obvious that this antenna array structure supports wireless applications in frequency range of $2.4 \mathrm{GHz}$. A typical antenna array configuration is shown in Figure 3.

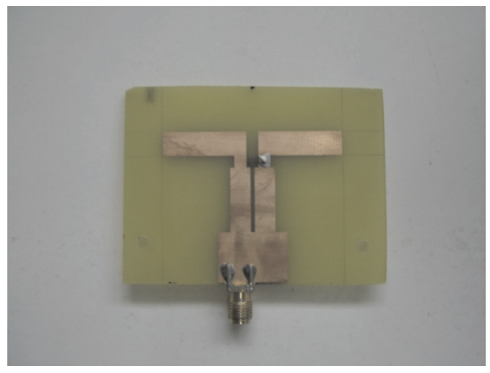

(a) Bottom Layer

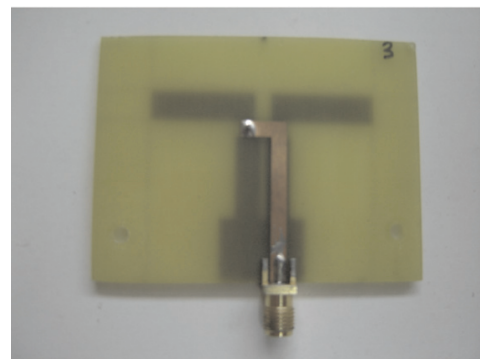

(b) Top Layer

Figure 1. Printed dipole antenna.

Table 1. Results for printed dipole (simulated/measured).

\begin{tabular}{cccc}
\hline Definition & Symbol & Simulated & Measured \\
\hline $\begin{array}{c}\text { Resonance Center } \\
\text { Frequency }\end{array}$ & $f_{0}(\mathrm{GHz})$ & 2.3 & 2.4 \\
Resonance Bandwidth & $B W(\mathrm{GHz})$ & 0.5 & 0.5 \\
Return Loss & $R L(\mathrm{~dB})$ & -58 & -42 \\
\hline
\end{tabular}

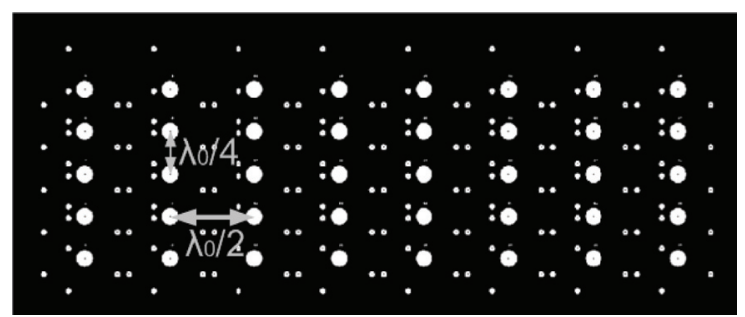

Figure 2. Top site of plane reflector.

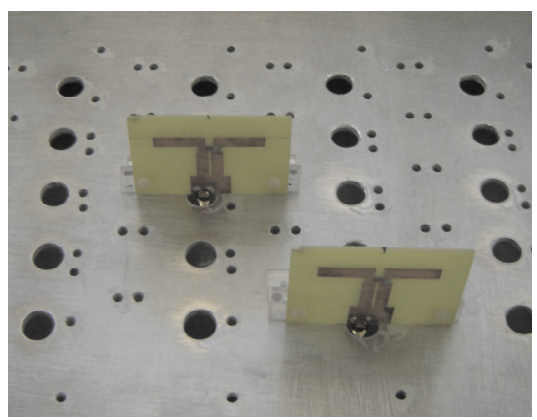

Figure 3. Typical antenna array configuration. 


\section{Analysis and Discussion}

The antenna system that has been introduced is studied and investigated in terms of envelope coefficient measurements. The corresponding results have been obtained in each case of eight antenna array configurations. The first four of them corresponds to side by side placement of identical printed dipoles and are grouped to the first part of the present investigation. The last four represent collinear form and provides the second part. Figure 4 and Figure 5 show the corresponding groups of antenna array configurations for each part of the proposed investigation.

The corresponding $S$-parameters that are provided by experimental procedure have been concentrated and used to the mathematical formula proposed by in Equation (2). These experimental measurements of scattering parameters have been provided by a Network Analyzer and have taken place in two Laboratory's Environments A and B. In each of the two indoor environments and for Group A and Group B antenna array configurations these parameters have been obtained. With these measured results we calculate the envelope correlation coefficient. As mentioned above, this procedure is based on in Equation (2).

The corresponding calculations provide envelope correlation values in a frequency range of $1 \mathrm{GHz}$ with center frequency of $2.4 \mathrm{GHz}$. The experimental results for Group A antenna array configurations are shown in Figure 6 and Figure 7 for two different Laboratory Environments $\mathrm{A}$ and $\mathrm{B}$, respectively.

In each case of Group A configurations the corresponding envelope correlation curve has been plotted. From Figure 6 and Figure 7, it seems that the " $\rho$ " parameter has almost small values in this frequency range. In particular, at frequency range of 1.5 to $2.1 \mathrm{GHz}$ the

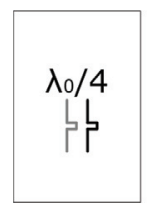

(a)

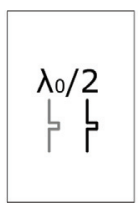

(b)

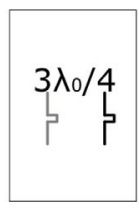

(c)

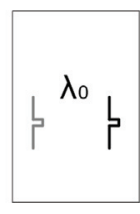

(d)
Figure 4. Relating antenna elements positions in the reflector backplane (Group A).

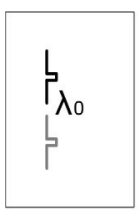

(e)

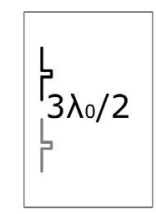

(f)

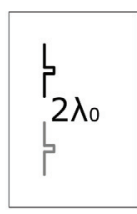

(g)

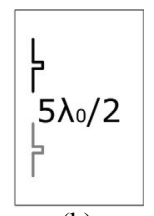

(h)
Figure 5. Relating antenna elements positions in the reflector backplane (Group B).

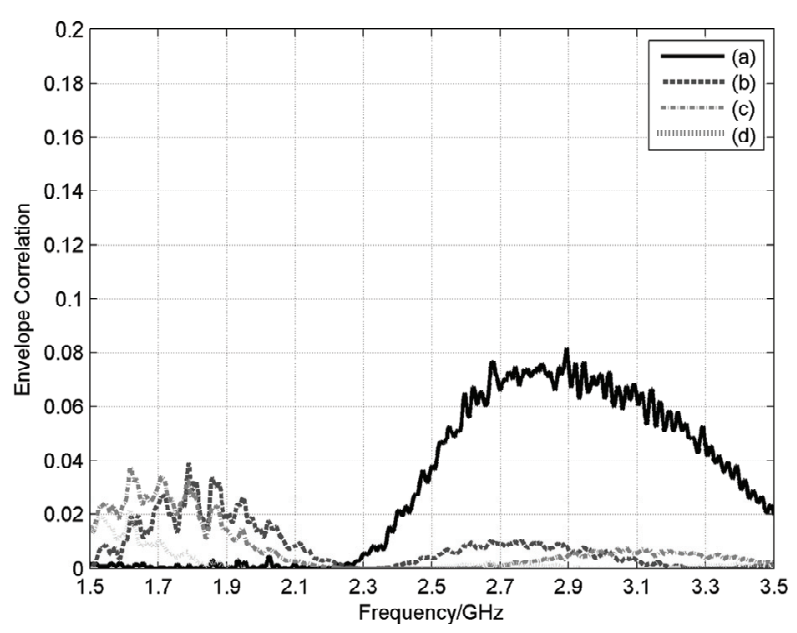

Figure 6. Measured results of envelope correlation for Group $A$ in Laboratory A environment.

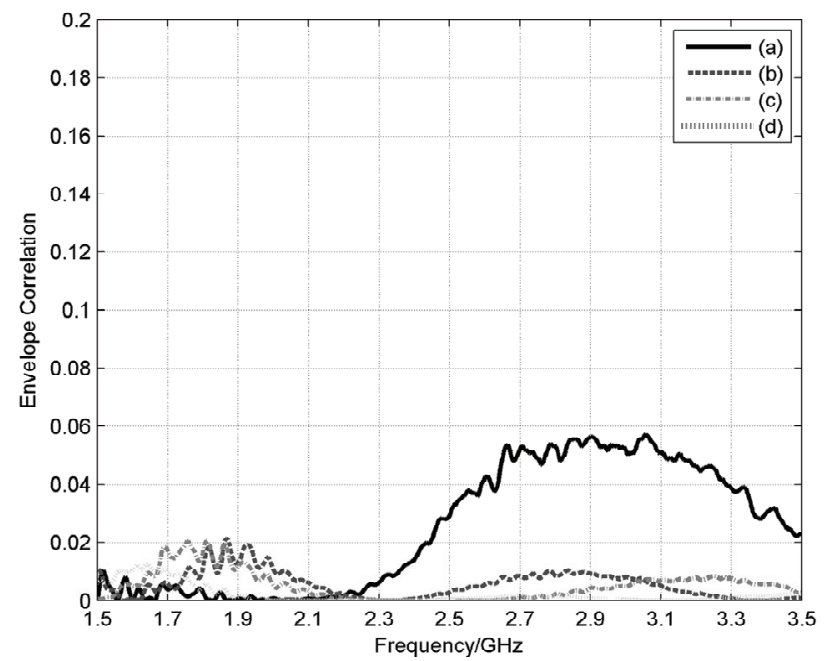

Figure 7. Measured results of envelope correlation for Group A in Laboratory B environment.

envelope correlation coefficient has a value close to 0.015 for all antenna array configurations in Group A. This observation indicates that the parameter " $\rho$ " remains stable as the distance between the dipoles increases in side by side antenna array configuration of Group A. Instead, at frequency range from 2.1 to $3.5 \mathrm{GHz}$ the value of envelope correlation coefficient has variations in a range of 0 to 0.05 for the smallest distance between the dipoles in Group A antenna array configuration. In other cases these variations are neglected.

For Group B the corresponding results for parameter " $\rho$ " are depicted in Figure 8 and Figure 9 for Laboratory's environment A and B, respectively.

From these experimental results, it seems that the parameter of envelope correlation has also quite small val- 
ues in the frequency range of 1.5 to $3.5 \mathrm{GHz}$. Small variations of this parameter is introduced at the frequency range from 1.5 to $2 \mathrm{GHz}$ only for the smallest distance between the dipoles in antenna array configuration of Group B. These variations are not provided by other values of distance between the elements bigger than one wavelength for collinear antenna array configuration. Moreover, in the frequency range from 2 to $3.5 \mathrm{GHz}$ the parameter of envelope correlation coefficient remains stable and close to zero for each of the collinear antenna array configuration in Group B.

It is also important that these considerations for group $\mathrm{A}$ and B antenna array configurations are valid for laboratory's environments $\mathrm{A}$ and $\mathrm{B}$. The corresponding declines between the two different propagation envi-

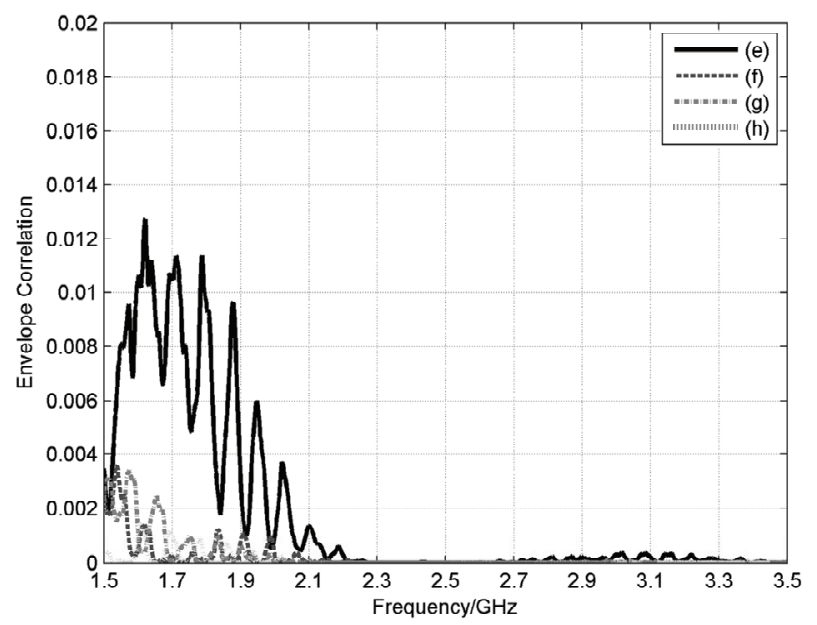

Figure 8. Measured results of envelope correlation for Group $B$ in Laboratory A environment.

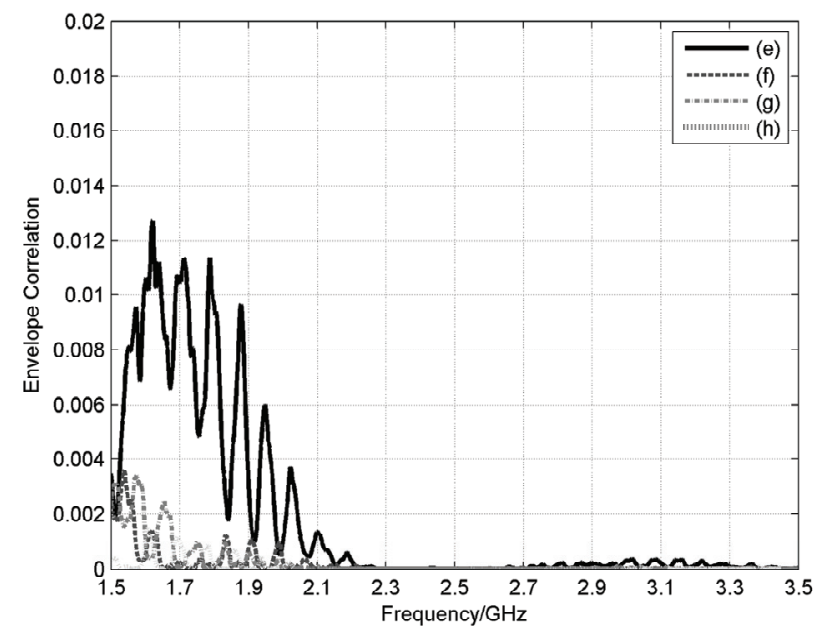

Figure 9. Measured results of envelope correlation for Group $B$ in Laboratory $B$ environment. ronments are neglected. In each of these indoor environments it is the rich multipath propagation procedure that provides this important agreement in the corresponding measured results. It is convenient that the indoor environments offer propagation processes with a big number of independent signal paths. This observation indicates that in rich multipath environments the corresponding signal paths are uncorrelated and are impossible to be in deep fade, simultaneously. This feature improves the performance of MIMO communication applications and systems.

Another important consideration is that in Group B antenna array configurations the envelope correlation is quite low relative to Group A. Different antenna array geometry may be an important issue for this effect. In any case of them, the value of envelope correlation is quite small and less of value 0.5 .

To sum up, these antenna array configurations provide low envelope correlation between the signals that have received or transmitted from the corresponding elements. In particular, Group B configurations have quite better correlation performance and offer more efficiently in MIMO applications' performance.

\section{Conclusions}

Antenna array configurations have been studied and investigated in terms of envelope correlation coefficient from the scattering parameters of two dipole antenna elements. The corresponding closed-form expression has been introduced and used to calculate this crucial parameter. This mathematical formula requires less laborious calculations and provides knowledge on antenna diversity optimization. Measured results indicate that rich multipath environment yields to low correlation coefficients for the proposed antenna array configurations. Several distances between antenna elements do not affect this parameter, dramatically. The corresponding considerations are very crucial for MIMO system's performance and are supported by an accurate method for investigation on modern wireless applications' design.

\section{Acknowledgment}

This research project (PENED) is co-financed by E.U. -European Social Fund (80\%) and the Greek Ministry of Development-GSRT (20\%).

\section{References}

[1] G. J. Foschini and M. J. Gans, "On Limits of Wireless Communications in a Fading Environment When Using Multiple Antennas," Wireless Personal Communications, Vol. 6, 1998, pp. 311-335. 
[2] R. Janaswamy, "Effects of Mutual Coupling on the Capacity of Fixed Length Linear Arrays," IEEE Antennas and Wireless Propagation Letters, Vol. 1, 2002, pp. 157-160.

[3] R. G. Vaughan and J. B. Andersen, "Antenna Diversity in Mobile Communications," IEEE Transactions on Vehicular Technology, Vol. 36, 1987, pp. 149-172.

[4] R. G. Vaughan, "Signals in Mobile Communications," IEEE Transactions on Vehicular Technology, Vol. 35, 1986, pp. 133-145.

[5] G. Lebrun, S. Spiteri and M. Faulkner, "MIMO Complexity Reduction through Antenna Selection," Proceedings on Australian Telecommun Cooperative Research Center, ANNAC'03, Vol. 5, 2003.

[6] S. Jacobs and C. P. Bean, "Fine Particles, Thin Films and Exchange Anisotropy," In Magnetism, G. T. Rado and H. Suhl, Eds., Academic, New York, Vol. 3, 1963, pp. 271-350.

[7] R. H. Clarke, "A Statistical Theory of Mibile Reception," Bell System Technology Journal, 1968, pp. 9571000. R. Nicole, "Title of Paper with Only First Word Capitalized," Journal Name Standard Abbreviations, in Press.

[8] K. Boyle, "Radiation Pattern and Correlation of Closely
Spaces Linear Antennas," IEEE Transactions on Antennas Propagation, Vol. 50, 2002, pp.1162-1165.

[9] H. T. Hui, W. T. OwYong and K. B. Toh, "Signals Correlation between Two Normal-Mode Helical Antennas for Diversity Reception in a Multipath Environment," IEEE Transactions on Antennas Propagation, 2004, pp. 572-577.

[10] J. Blanch, J. Romeu and I. Cordella, "Exact Representation of Antenna System Diversity Performance from Input Parameter Description," Electronics Letters, Vol. 39, 2003, pp. 705-707.

[11] H.-R. Chuang and L.-C. Kuo, "3-D FDTD Design Analysis of a $2.4 \mathrm{GHz}$ Polarization - Diversity Printed Dipole Antenna with Integrated Balun and Polarization - Switching Circuit for Wlan and Wireless Communication Application," IEEE Transactions on Microwave Theory and Techniques, Vol. 51, No. 2, 2003.

[12] Z. Fan, L. Ran and K. Chen, "Printed Dipole Antenna Designed with Microstrip Balun on V-Shaped Ground Plane," Progress in Electromagnetics Research Symposium Hangzhou, 2005, pp. 23-26.

[13] D. M. Pozar, "Microwave Engineering,” Wiley, 1998. 